\title{
STUDIES ON THE EFFECTS OF HYPERBARIC OXYGENATION ON THE OPSONIC INDEX AND HAEMATOLOGICAL BLOOD IMAGE IN RABBITS
}

\author{
Tadeusz Doboszyński, Kazimierz Ulewicz, Bogdan Łokucijewski, Przemysław Michniewski
}

Department of Maritime Medicine of the Military Medical Academy in Gdynia, Poland

\section{ABSTRACT}

Hyperbaric oxygen toxicity studies were conducted on rabbits using the opsonic index determination. The study was conducted on 15 animals that had opsonin index examined prior to hyperbaric oxygen exposure. They were then subjected to an hourly exposure to hyperbaric oxygen with overpressure values of $1.8,2.4$ and $3.1 \mathrm{~atm}$ in groups of 5 animals. After the exposure, the opsonium index was re-examined upon the lapse of 1,2 and 10 days. Parallelly, the morphological image of the blood was examined.There was a statistically significant increase in the index in the first two days after exposure, independent of the value of oxygen overpressure. On the 10th day, the index value approached the initial one.

Keywords: hiperbaric, oxygen, toxicity, opsonins

ARTICLE INFO

PolHypRes 2018 Vol. 63 Issue 2 pp. 45 - 50

ISSN: 1734-7009 eISSN: 2084-0535

\section{Original article}

DOI: $10.2478 /$ phr-2018-0012

Originally published in Bulletin of the Military Medical

Pages: 6, figures: 0 , tables: 2

Academy 1967 r.

page www of the periodical: www.phr.net.pl

Date of approval for print in PolHyp Res: 09.03.2017 r.

Publisher

Polish Hyperbaric Medicine and Technology Society 


\section{INTRODUCTION}

The problems regarding the effects of hyperbaric oxygenation and oxygen toxicity on experimental animals and humans, as well as on the course of the immunological reactions in these conditions, have already been the subject of our interest $[13,4,5,8,10]$ and now prompt us to aim at determination of oxygen toxicity based on the opsonic index. In contrast to a number of publications on the changes in the phagocytic index as an expression of systemic reactivity to toxic agents $[9,11]$, no data have been found in the available literature on the use of the opsonic index in the determination of the effects of hyperbaric oxygenation on the body. The application of the opsonic index for this type of research seemed intentional due to the possibility of eliminating leukocytes from the experimental animals used in the study, thus avoiding the direct impact on their level during hyperbaric oxygenation.

The choice of the immunological method was also determined by the fact that hyperbaric oxygenation affects the activity of the pituitary-adrenal cortex axis $[1,6,2]$, which in turn affects the processes of immunogenesis [3]. These processes are stimulated by mineralocorticoids and are expressed by an increase in the phagocytic activity, which confirms the possibility of suppression of stimulation and thus of the phagocytic leukocyte activity in the blood by eliminating the adrenal cortex [12]. These data emphasise the role of the endocrine system in immune processes, similarly as it is observed in the nervous system [3], in the processes of regulation of synthesis of antibodies.

\section{MATERIAL AND METHOD}

The study was conducted on adult rabbits of both sexes, with a weight of between $2.0-2.5 \mathrm{~kg}$, bred in standard conditions. In the first phase of tests (15 animals), the opsonic index was determined according to the method developed by Zabłocki (16) using guinea pig leukocytes and a haemolytic (haemolysis alpha), enterotoxigenic A (enterotoxin A), coagulase positive staphylococcus strain number 264 using a bacterial suspension with a concentration of approximately 300 million microorganisms in $1 \mathrm{ml}^{3}$. Next, the animals underwent hyperbaric oxygenation at a pressure of 1.8 atm, $2.4 \mathrm{~atm}, 3.1 \mathrm{~atm} \mathrm{O}^{2}$, with given overpressures applied in the chamber for one hour ( 5 animals were used for each pressure). Subsequently, the opsonic index in the blood samples taken prior to the exposure was determined in each animal one hour after the exposure, 24 and 48 hours after the exposure, as well as upon the lapse of 10 days.

In parallel to these tests, haematological blood tests (haemoglobin, erythrocytes, leukocytes, index, reticulocytes and haematocrit) were performed, as well as the division of proteins in the blood serum of the tested rabbits using paper electrophoresis, the KWE-3 apparatus and medicinal buffer.

\section{RESULTS}

The obtained results on the opsonic index in animals subjected to hyperbaric conditions are illustrated in Table 1

The analysis of the results presented in this table, prepared using the student test for verifying the statistical significance with $\mathrm{P}=99.9$, revealed a statistical significance of results of the opsonic index for the control group $(\mathrm{x})=0.6$ and animals tested following the exposure to hyperbaric oxygen at 1.8, 2.4 and 3.1 atm upon the lapse of 1 one hour, 24 hours, 48 hours and ten days from the exposure.

The narrow scope of materials regarding haematological tests prompted a collective representation of results obtained for pressures from 1.8 to $3.1 \mathrm{~atm}$. The data are presented in Table 2. 


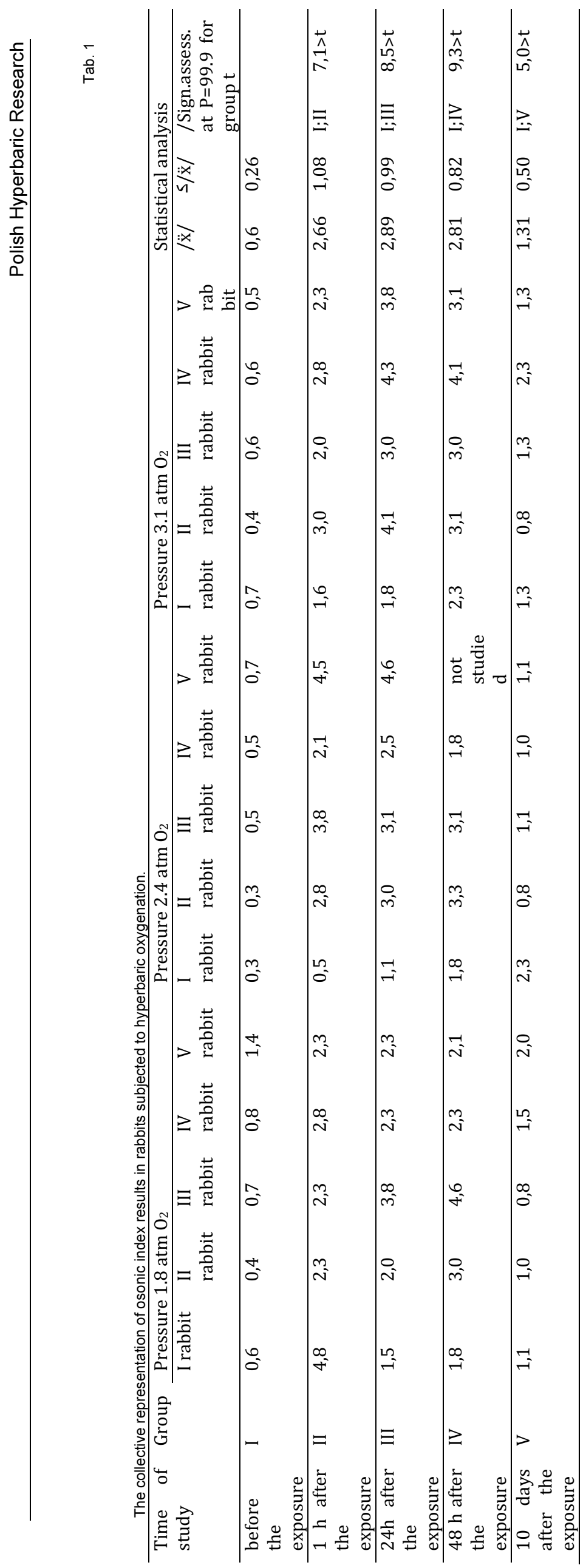




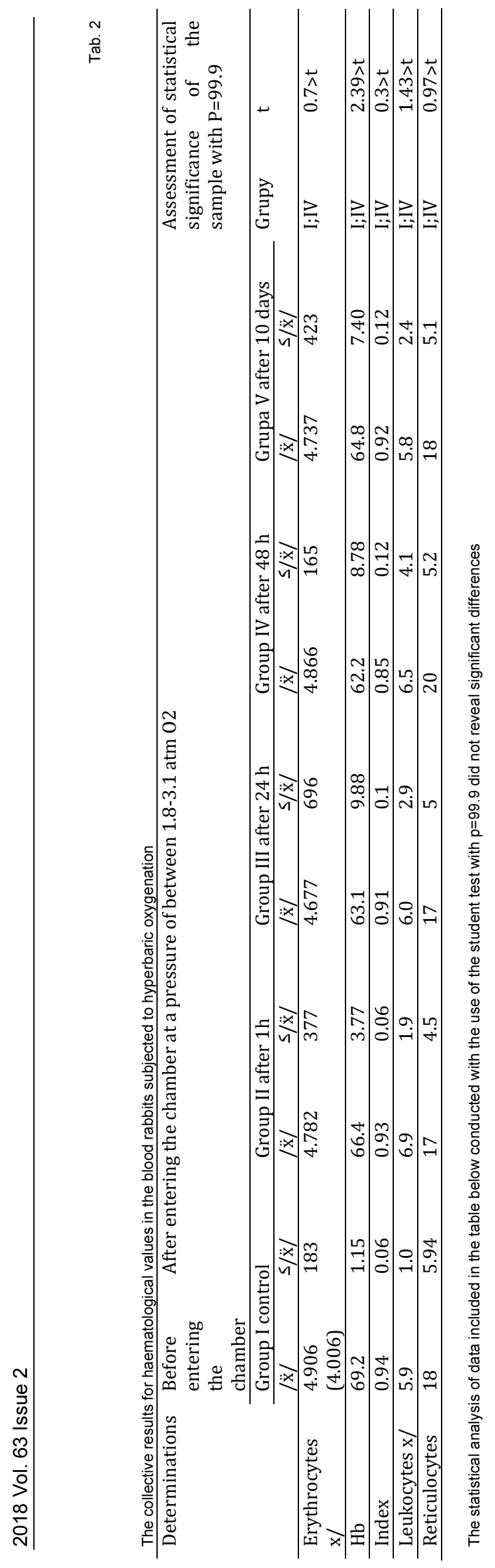




\section{DISCUSSION AND RESULTS}

The presented table shows that in "normal" rabbits the opsonic index fluctuated within a range of between 0.3 and 1.4 (the average of 0.6 ). In only one case it reached a value of 1.4. In all animals treated with hyperbaric oxygenation (at $1.8 \mathrm{~atm}, 2.4 \mathrm{~atm}$, and $3.1 \mathrm{~atm}$ ) an increase of the opsonic index was observed, at times even as high as 4.8 , with no significant differences seen either in the level of the index or the time taken to reach its increased level, depending on the period of blood sampling and the oxygen partial pressure. In most cases, the opsonic index tended to normalise after 10 days.

As can be seen from the above-mentioned data, there were no significant shifts in the haematological blood image in rabbits subjected to hyperbaric oxygenation. In fact, the observed changes are within the limits of fluctuations in the values obtained in "normal" animals.

Similarly, in the electrophoretic studies on the serum of the tested animals, no significant differences were found in individual fractions in the same animals before and after the experiment, as well as in subsequent periods of observation. The observed differences were not permanent and did not exceed the error of the method.

From the data quoted above, it would appear that hyperbaric oxygenation within the range between 1.8 and $3.1 \mathrm{~atm} . \mathrm{O}^{2}$ increases the opsonic index, which may be an expression of a toxic effect of oxygen on the animal system, however on the basis of the presented results it is difficult to say what the mechanism of this phenomenon is. It seems that both immunological mechanisms $[15,14,7]$, and the mechanisms within the pituitaryadrenal cortex axis [3] could be involved, as well as the mechanism of action conditioned by the presence of additional metabolites [11], or a combined effect of these mechanisms. In any case, further research in this direction is necessary to fully investigate this phenomenon. Finally, it should be added that in our opinion the use of the opsonic index in the studies on the effects of hyperbaric oxygenation on the body is promising, however, it requires additional testing on a larger material and under varied experimental conditions (including in vitro studies with the use of a tissue culture - similar to 17).

\section{REFERENCES}

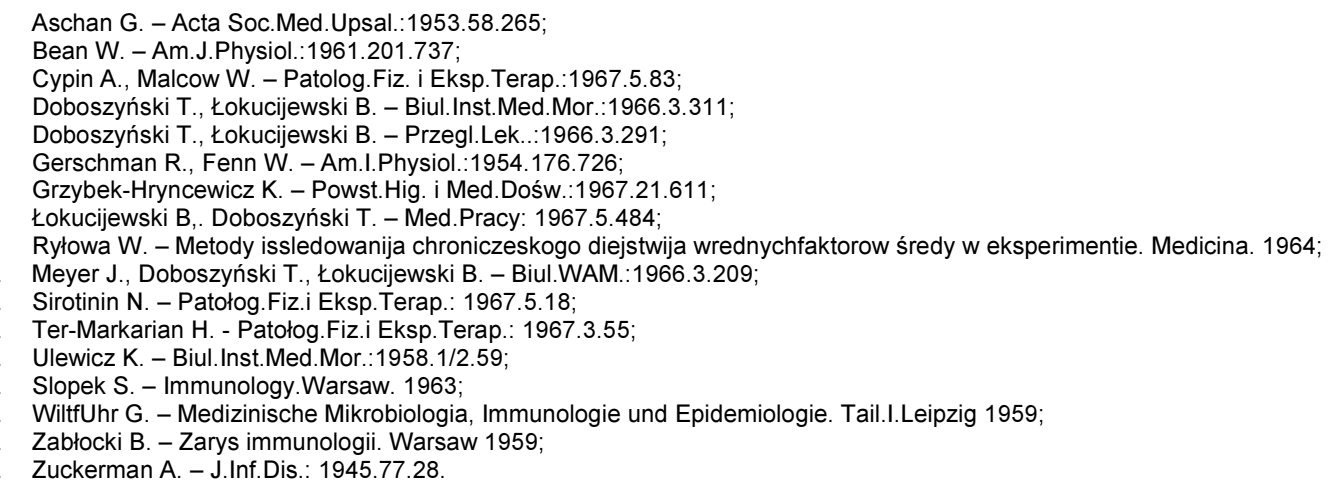

